\title{
OGT-mediated O-GIcNAcylation on GLDC promotes metastasis in cervical cancer
}

\author{
Pingsheng Cai ${ }^{2, *}$, Xuejing Jin ${ }^{2,4, *}$, Hua Zhu ${ }^{3}$, Shupin Zhu ${ }^{2}$, Lijie Wang ${ }^{2}$ and Jing Jin ${ }^{1}$ \\ ${ }^{1}$ Institute of Glycobiological Engineering, Zhejiang Provincial Key Laboratory of Medical Genetics, Wenzhou Medical University, \\ Zhejiang, China \\ ${ }^{2}$ Department of Obstetrics and Gynecology, Wenzhou Hospital of Integrated Traditional Chinese and Western Medicine, \\ Wenzhou, Zhejiang, China \\ ${ }^{3}$ Department of Obstetrics and Gynecology, The First Affiliated Hospital of Wenzhou Medical University, Wenzhou, Zhejiang, \\ China \\ ${ }^{4}$ Present address: Hangzhou Women's Hospital (Hangzhou Maternity and Child Health Care Hospital), Hangzhou, China \\ *These authors share co-first authorship \\ Correspondence to: Jing Jin, email: jinjing@wmu.edu.cn \\ Keywords: glycine decarboxylase (GLDC); O-GlcNAcylation; OGT; cervical cancer; metastasis \\ Received: November 27, $2017 \quad$ Accepted: January 03, $2018 \quad$ Published: January 09, 2018 \\ Copyright: Cai et al. This is an open-access article distributed under the terms of the Creative Commons Attribution License 3.0 \\ (CC BY 3.0), which permits unrestricted use, distribution, and reproduction in any medium, provided the original author and source \\ are credited.
}

\section{ABSTRACT}

Glycine decarboxylase (GLDC) is one of the glycine metabolic enzymes and was reported as a oncogene involved in tumorigenesis of non-small cell lung cancer. However, the function of GLDC in cervical cancer remains unclear. In this study, we determined the increased expression of GLDC in cervical cancer tissues and cell lines. The clinical pathological characters of GLDC revealed that increased GLDC expression was involved in cervical cancer metastasis. Further in vitro experiments confirmed the promotion of GLDC on cervical cancer metastasis. Furthermore, we found that OGT interacted with GLDC and modified GLDC with O-GIcNAcylation in cervical cancer cell lines. In addition, the O-GICNAcylation of GLDC greatly enhanced its promotion on migration and invasion in vitro and in vivo. The clinical data further demonstrated the promotion of GLDC and OGT on cervical cancer metastasis, and showed that OGT worsened the prognosis of GLDC ${ }^{\text {High }}$ patients. Collectively, this study identified OGT-mediated O-GICNAcylation on GLDC as an essential regulatory mechanism for promoting cervical cancer metastasis, and provided a therapeutic target for metastatic cervical cancer.

\section{INTRODUCTION}

Cervical cancer is the fourth most prevalent type of cancer among women worldwide, which accounts for $9 \%$ $(n=527,600)$ of incident cases of cancer and $8 \%(n=$ $265,700)$ of total cancer mortality in 2012 [1]. Although the development of early screening and HPV vaccination reduces the mortality rates in recent years $[2,3]$, the prognosis of patients is still dismal, mainly due to the high incidence of invasiveness and metastasis of cervical cancer [4]. Therefore, investigating the molecular alterations that drive metastasis in cervical cancer is valuable to develop diagnostic targets and potential therapies for this disease.
Glycine dehydrogenase (GLDC) is a metabolic enzyme involved in glycine and serine metabolism, which catalyzes the reaction whereby glycine is converted to carbon dioxide, ammonia and 5,10-methylenetetrahydrofolate (CH2-THF) [5-7]. In turn, $\mathrm{CH} 2-\mathrm{THF}$ drives de novo thymidine synthesis and pyrimidine biosynthesis, thus regulating nucleotide synthesis during cell proliferation [8]. GLDC has been reported as a metabolic oncogene involved in tumorigenesis of nonsmall cell lung cancer (NSCLC) and was found to be critical in tumor initiating cell growth and tumor formation [9]. Actually, besides of NSCLC, the increased expression of GLDC was also observed in some other cancers 
including ovarian cancer, seminoma, invasive lobular carcinoma, and breast cancer [9-11]. And the increased expression of GLDC was reported to be associated with the poor prognosis of phyllodes tumor, thyroid cancer, invasive lobular carcinoma, and metastatic breast cancer [11-14]. However, the roles of GLDC in cervical cancer have never been determined.

O-GlcNAcylation is a reversible posttranslational modification on various proteins implicating in different cellular functions [15-18], and increased O-GlcNAcylation were observed in various cancers due to the elevated glucose flux through hexosamine biosynthetic pathway $[19,20]$. In turn, increased O-GlcNAcylation promotes oncogenesis and tumour growth, as well as metastasis in different cancers [21-24]. Recently, O-GlcNAcylation and its transferase O-linked $\mathrm{N}$-acetylglucosamine transferase (OGT) were reported to be involved in cervical cancer tumorigenesis and metastasis via increasing or activating the human papillomavirus (HPV)-derived oncoproteins [25, 26]. These studies also showed that the O-GlcNAcylation on some oncoproteins such as c-MYC and HCF-1 elevated their functional activities $[25,26]$. Whether O-GlcNAcylation confers similar functions on other oncoproteins in cervical cancer remains unclear.

In the present study, we assessed the expression of GLDC in cervical cancer, and investigated the roles of GLDC in cervical cancer in vitro and in vivo. We found that OGT-mediated O-GlcNAcylation on GLDC promotes metastasis in cervical cancer. Additionally, we determined the clinical significance of the O-GlcNAcylation on GLDC as well as its correlation with cervical cancer metastasis.

\section{RESULTS}

\section{GLDC expression is increased in cervical cancer}

To determine the expression of GLDC in cervical cancer, we performed qRT-PCR, WB and IHC analysis of 146 paired cervical cancer tumor and non-tumor samples. Results showed that the expression of GLDC was significantly increased in cancer tissues compared with normal tissues in both mRNA $(p=0.0421)$ and proteins levels ( $p=0.0138$ in $\mathrm{WB}$, and $p=0.0002$ in IHC) (Figure 1A-1C). WB and qRT-PCR assays were also performed in different cervical cell lines. Consist with tissues, cervical cancer cell lines (HT-3, C33A, Caski and HeLa) conferred significantly higher levels of GLDC compared with the normal cervix cells (End1) (Figure 1D-1E). Similar results were also observed in public cervical cancer databases $(P=0.0089$ in GSE27678, and $p=0.0371$ in GSE52903) (Figure 1F). Together, these data indicate that GLDC expression is increased in cervical cancer.

\section{Increased GLDC expression in cervical cancer cells promotes metastasis}

We further analyzed the relationship between GLDC and clinical pathological characteristics of cervical cancer in IHC analysis. All cervical cancer specimens were grouped according to their age, FIGO stage, tumor grade, tumor size and lymph node metastasis. The statistic results showed that increased GLDC expression in cervical cancer was significantly correlated with tumor grade $(P=0.002)$ and lymph node metastasis $(P=0.027)$ (Table 1), suggesting that GLDC may be involved in cervical cancer metastasis. To confirm the function of GLDC in cervical cancer cells, we made an optimal dynamic range of GLDC expression in different cells. GLDC was knockdown in Hela cells, which have relatively high endogenous GLDC levels, and GLDC was overexpressed in HT-3 cells, which have relatively low levels of endogenous GLDC (Figure 2A). Utilizing these cells in transwell and scratch assays, we found that the knockdown of GLDC inhibited cell migration $(P<$ $0.05)$ and invasion $(P<0.01)$, while the overexpression of GLDC significantly promoted the migration $(P<0.001)$ and invasion $(P<0.05)$ of cervical cancer cells (Figure 2B-2C). However, although the expression level of GLDC in $\mathrm{C} 33 \mathrm{~A}$ cells was as high as that in Hela cells (Figure $1 \mathrm{D}-1 \mathrm{E})$, the migration and invasion of C33A cells were significantly weaker than Hela cells (Figure 2D-2E), suggesting that something in $\mathrm{C} 33 \mathrm{~A}$ cells regulates the effects of GLDC on metastasis.

\section{OGT interacts with and modifies GLDC with O-GlcNAcylation}

Post-translational modifications have been reported to regulate the functions of some glycine/serine metabolism enzymes such as SHMT1 and MTHFR [27-29]. However, on another glycine/serine metabolism enzyme GLDC, no post-translational modification has been reported. Since similar GLDC levels resulted in different effects on metastasis in different cell lines (Figure 2D-2E), we hypothesized that GLDC might be modified in some cell lines and the potential modifications on GLDC could regulate its effects on migration and invasion. Different from SHMT1 or MTHFR [27-29], there was no phosphorylation detected on GLDC in our study (Figure 3A). But we observed the O-GlcNAcylation on GLDC for the first time (Figure 3A). Although the O-GlcNAcylation of GLDC was detected in both Hela and C33A cells, the O-GlcNAcylation level of GLDC in Hela cells was markedly higher than that in C33A cells (Figure $3 \mathrm{~A})$. These results, combined with the in vitro metastasis data of different cell lines (Figure 2D-2E), suggest that O-GlcNAcylation on GLDC might be the key role for promoting cervical cancer metastasis. 
Since O-GlcNAcylation is regulated by OGT and OGA, we also assessed the protein levels of OGT and OGA in Hela and C33A cells, and found that OGT expression varied a lot between these two cell lines, while no obvious difference of OGA expression was observed (Figure 3A). Meanwhile, coIP analysis of either transfected or endogenous proteins from cervical cancer cells confirmed the interaction between GLDC and OGT (Figure 3A-3B and 3D). And the direct interaction between these two proteins was determined by GST pull-down assay (Figure 3C). To further verify that the O-GlcNAcylation changes on GLDC are regulated by OGT, we detected the O-GlcNAcylation changes of GLDC in C33A cells with OGT overexpression or in Hela cells treated with the enzymatic inhibitors ST078925 or ST045849 against OGT. We found that OGT and its enzymatic activity were required to activate the O-GlcNAcylation of GLDC (Figure 3D-3E). Taken together, these results reveal that OGT could directly interact with GLDC, and modify the O-GlcNAcylation on GLDC.

\section{OGT enhances the promoting functions of GLDC on cervical cancer metastasis}

In order to demonstrate the promoting roles of the O-GlcNAcylation on GLDC in cervical cancer metastasis,
A

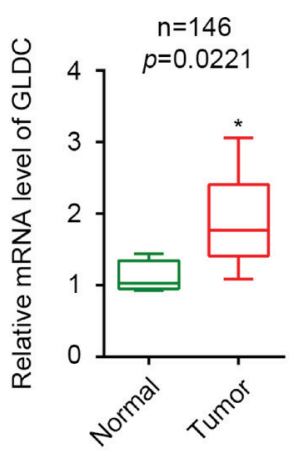

B
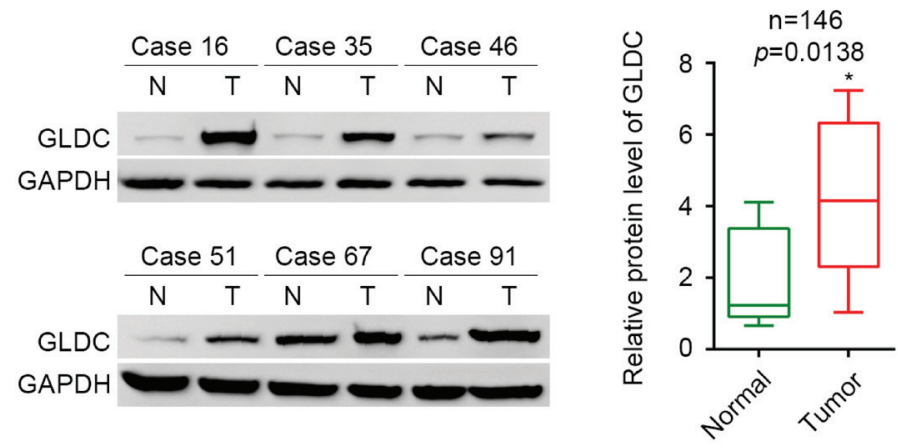

C
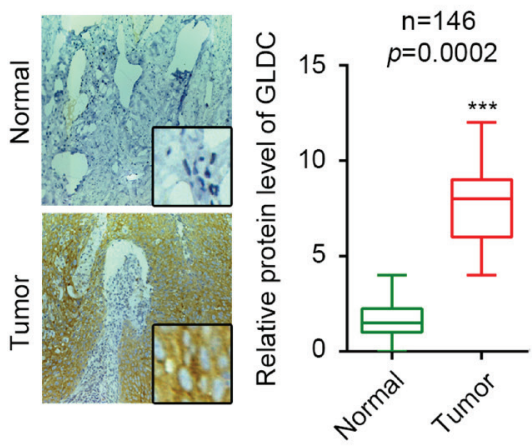

D

$E$

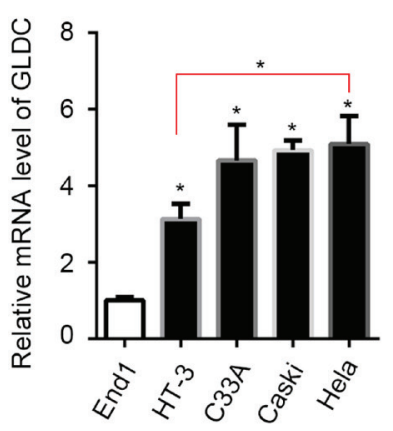

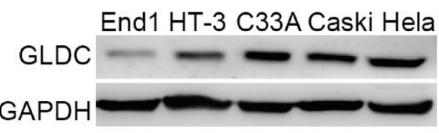

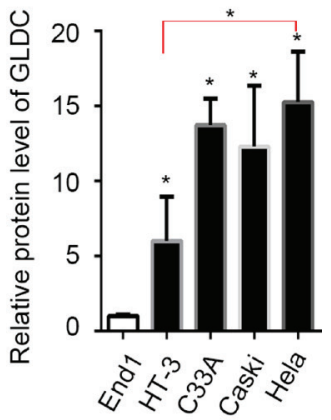

F

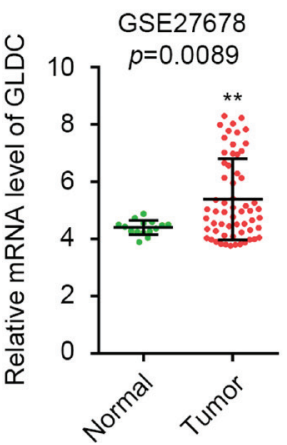

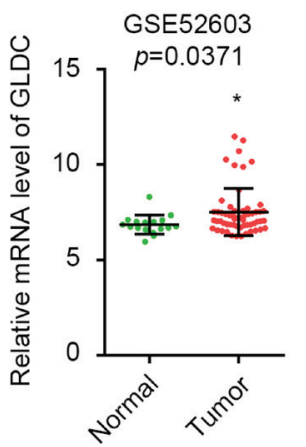

Figure 1: GLDC expression is increased in cervical cancer. (A) Relative mRNA levels of GLDC in 146 paired cervical cancer tumor and non-tumor samples were determined by qRT-PCR assays. (B-C) Protein levels of these tissues samples were determined by WB and IHC analysis. (D) Relative protein levels of GLDC were determined by qRT-PCR in a normal cervix cell line (End1) and in four cervical cancer cell lines (HT-3, C33A, Caski and HeLa). (E) mRNA levels of GLDC in End1, HT-3, C33A, Caski and HeLa cell lines were determined by qPCR. (F) The relative mRNA levels of GLDC were analyzed in public cervical cancer databases GSE27678 and GSE52903. For WB and IHC analysis, representative images (left) and statistical data (right) were shown. GAPDH was used as loading control in WB. ${ }^{*} P<0.05 ;{ }^{* *} P<0.01 ;{ }^{* * *} P<0.001$. 
Table 1: The correlation between GLDC expression and clinicopathologic characteristics in IHC analysis

\begin{tabular}{|c|c|c|c|}
\hline \multirow{2}{*}{ Clinical variable } & \multicolumn{3}{|c|}{ Relative expression $^{\mathrm{a}}$ of GLDC and $p$-value ${ }^{\mathrm{b}}$} \\
\hline & $N$ & Median & $p$-value \\
\hline Age & & & 0.633 \\
\hline$<45$ & 69 & 6.87 & \\
\hline$\geq 45$ & 77 & 6.94 & \\
\hline Tumor size & & & 0.112 \\
\hline$<5 \mathrm{~cm}$ & 70 & 6.29 & \\
\hline$\geq 5 \mathrm{~cm}$ & 75 & 7.03 & \\
\hline Grade & & & 0.002 \\
\hline $\mathrm{I}+\mathrm{II}$ & 92 & 4.03 & \\
\hline III & 54 & 9.96 & \\
\hline LN metastasis & & & 0.027 \\
\hline Yes & 59 & 9.37 & \\
\hline No & 84 & 4.65 & \\
\hline FIGO stage & & & 0.257 \\
\hline I-II & 50 & 6.41 & \\
\hline III-IV & 96 & 6.72 & \\
\hline
\end{tabular}

${ }^{a}$ Median of relative expression.

${ }^{b}$ Pearson $\chi^{2}$ test were used for comparing different groups.

A

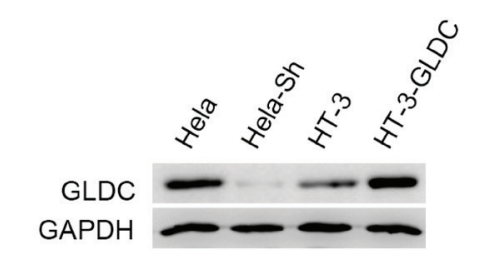

C

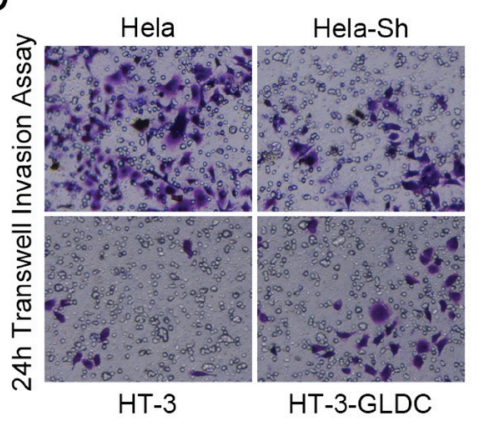

B

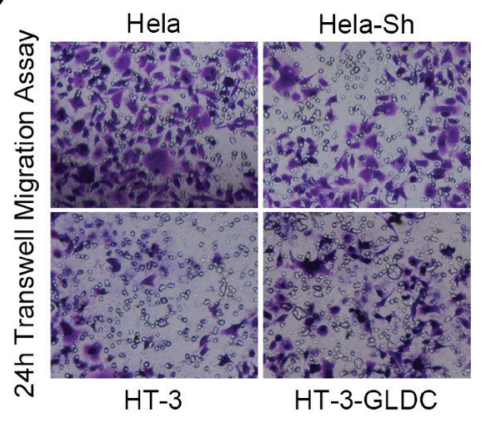

D

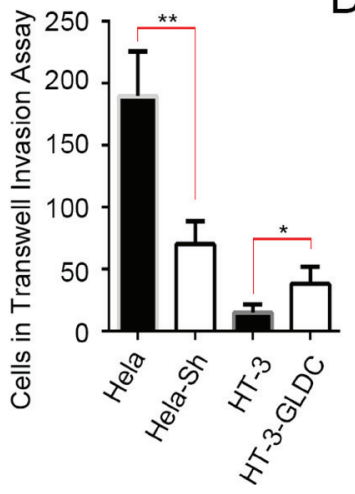

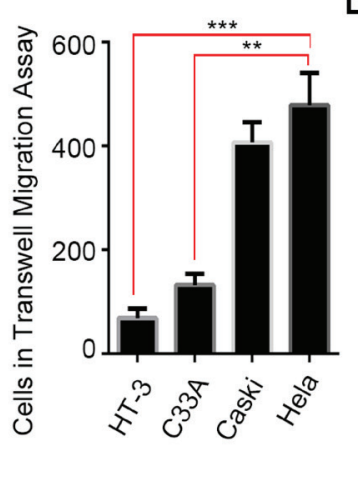

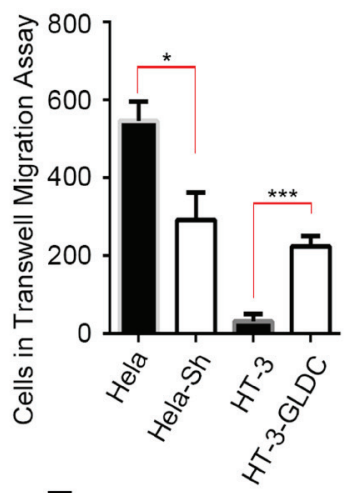

$E$

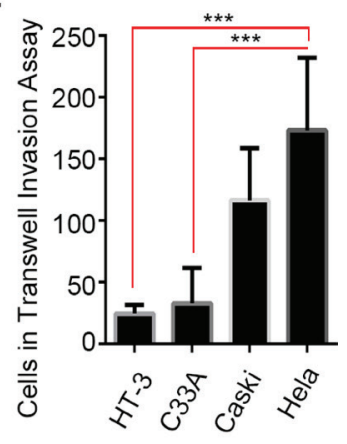

Figure 2: Increased GLDC expression in cervical cancer cells promotes metastasis. (A) Western blot of GLDC protein levels in Hela cells subjected to GLDC knockdown (Hela-Sh cells) and in HT-3 cells subjected to GLDC overexpression (HT-3-GLDC cells). (B-C) $24 \mathrm{~h}$ transwell migration and invasion assays in different cervical cancer cells (Hela, Hela-Sh, HT-3 and HT-3-GLDC). Representative images (left) and statistical data (right) were shown. (D-E) $24 \mathrm{~h}$ transwell migration and invasion assays in HT-3, C33A, Caski and HeLa cells. Statistical data were shown. All experiments were repeated more than 3 times. ${ }^{*} P<0.05 ;{ }^{* *} P<0.01 ;{ }^{* * *} P<0.001$. 
we took advantage of OGT enzyme-activity inhibitors and different cervical cancer cells subjected to overexpression or knockdown of GLDC and/ or OGT. In vitro migration data revealed that the increased expression of OGT in GLDC $^{\text {High }}$ cells (C33A-OGT, Hela and HT-3-GLDC cells) greatly enhanced the promotion of GLDC on migration, and the inhibition of OGT enzyme activity in GLDC ${ }^{\text {High }}$ cells significantly decreased migrated cell numbers, while the inhibition of OGT resulted in no significant changes on migration of GLDC ${ }^{\text {Low }}$ cells (C33A-ShGLDC, HelaShGLDC and HT-3 cells) (Figure 4A-4C). These data indicated that OGT and O-GlcNAcylation were capable to enhance the promotion of GLDC on metastasis. Actually, the negligible effect of OGT inhibitors in GLDC $^{\text {Low }}$ cells also suggested that the effects of OGT on metastasis somehow depended on the expression of GLDC in cervical cancer. The functional enhancement of OGT upon GLDC was further confirmed by in vivo metastasis result of intravenous injection and bioluminescent imaging (Figure 4D-4E).

\section{Co-expression of OGT and GLDC clinically correlates with cervical cancer metastasis and predicts poor prognosis in patients}

We next assessed the expression of OGT in 146 cervical cancer patients and evaluated the clinicopathological correlations of OGT levels with cervical cancer metastasis via immunohistochemistry analysis (Figure 5A-5B). We found that high levels of GLDC $(P=0.027)$ instead of OGT $(P=0.063)$ were positively associated with metastasis, and OGT significantly aggravated metastasis in GLDC ${ }^{\text {High }}$ patients $(P=0.008)$ (Figure 5B). Kaplan-Meier analysis and logrank tests were further used to investigate the prognostic value of OGT and GLDC expression on patient survival. The log-rank test results showed that the increased expression of GLDC $(P=0.0325)$ instead of OGT $(P=$ $0.0741)$ strongly correlated with poor prognosis in cervical cancer patients (Figure 5C), and patients with the overexpression of both two proteins had a worse prognosis compared with other GLDC ${ }^{\text {High }}$ patients $(P=$ 0.004) (Figure 5D), strongly supporting that OGT and O-GlcNAcylation enhanced the effects of GLDC in cervical cancer. These data also suggest that the O-GlcNAcylation on GLDC could be used as an factor that predicts poor prognosis in patients with cervical cancer.

\section{DISCUSSION}

GLDC is one of the glycine metabolic enzymes and was reported as a oncogene involved in tumorigenesis of NSCLC [9, 30]. Although some subsequent studies supported the tumor-promoting effects of GLDC in different cancers such as phyllodes tumor and thyroid cancer $[10,12]$, a recent study provided contradictory evidences for the role of GLDC in gastric cancer, which suggested GLDC might also function as tumor
A

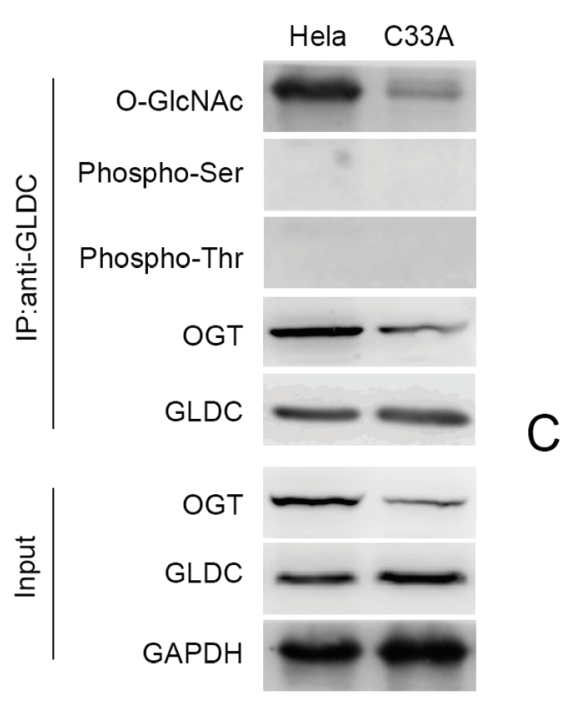

B

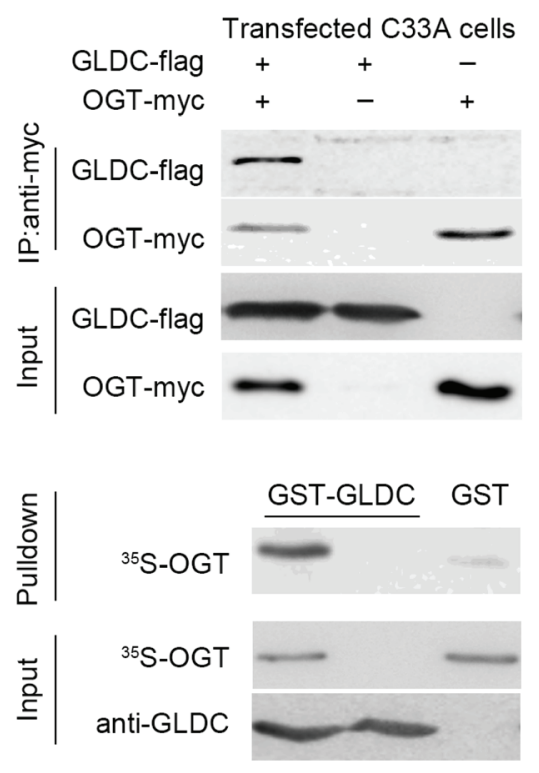

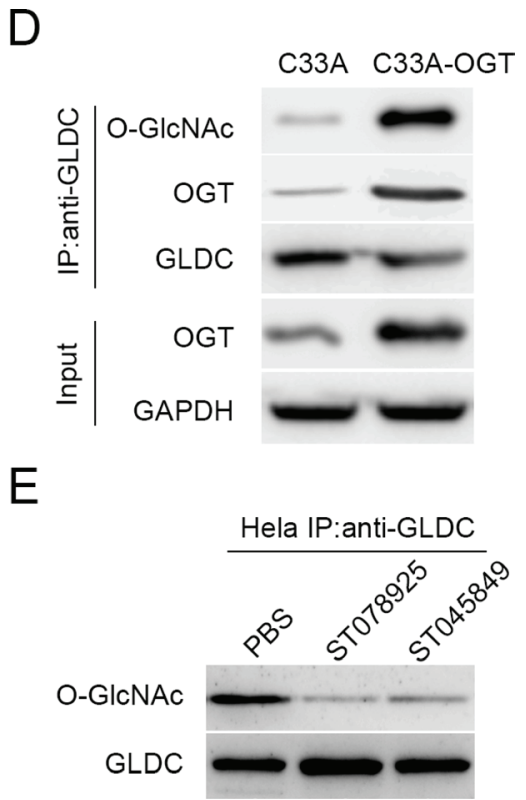

Figure 3: OGT interacts with and modifies GLDC with O-GIcNAcylation. (A) Protein levels of OGT and GLDC in cell lysate of Hela cells and C33A cells determined by WB. Interaction between GLDC and OGT, and post-translational modifications of GLDC determined by immunoprecipitation of GLDC from Hela cells and C33A cells. (B) Interaction between GLDC and OGT determined by immunoprecipitation of OGT from C33A cell co-transfected with GLDC-myc and GLDC-Flag. (C) Direct interaction between GLDC and OGT determined by GST pull-down assay. (D-E) Immunoprecipitation analysis of O-GlcNAcylation changes on GLDC. C33A cells subjected to OGT overexpression (D) or Hela cells treated with enzymatic inhibitors ST078925 (50 $\mu \mathrm{M})$ or ST045849 (50 nM) targeting OGT (E) were used. GAPDH was used as loading control. 
suppressor [31]. Therefore, in spite of the novel increased expression of GLDC in various cancers [9, 11, 31-33], the functions of GLDC in different kinds of tumors still remain unclear. In present study, we found that increased GLDC expression was detected in several different cervical cancer cell lines compared with a normal cervix cell line, which is consistent with the results reported in previous study. Moreover, our data show that this increased expression markedly promoted migration of cervical cancer cells, suggesting that GLDC is involved in metastasis promotion. Since GLDC functioned in driving tumorigenesis in cancer stem cells (CSCs) [9], and CSCs is critical for tumor metastasis [34-36], GLDC might be the connection between CSCs and metastasis. In one hand, metastasis could be triggered by CSCs via elevated activities of glycine metabolism enzymes including GLDC. In another hand, increased proportion of CSCs in primary tumor tissues mediated by increased GLDC would also promote metastasis. Actually, similar with GLDC, some other glycine/serine metabolism enzymes such as SHMT1/2, PSPH, and PSAT1, are involved in both tumorigenesis [9] and metastasis [14, 37, 38]. And increased expression of these enzymes in tumors predict poor prognosis [38, 39]. These observations provide evidences for the notion that metabolic reprogramming, especially glycine/serine metabolic reprogramming in cancer cells might be crucial for metastasis [40].

Post-translational modification is critical for various biological functions of almost all known proteins, including signal transduction, degradation, polymerization, as well as activation, especially enzymatic activation. In glycine metabolism, the enzymatic activities of several enzymes could be regulated by post-translational modifications. For instance, phosphorylation of SHMT1 was reported to play potential roles in cancer-modulating property of p38 MAP kinase [27], and phosphorylation of MTHFR enhanced the activity of MTHFR and promoted heterochromatin maintenance [28, 29]. As for another glycine metabolic enzyme GLDC, there is no modification on it reported before. In this study, our data reveal the O-GlcNAcylation on GLDC for the first time, and demonstrate that this modification is involved in the functions of GLDC on cervical cancer metastasis.

Actually, O-GlcNAc is another link between cancer cell metabolic reprogramming and metastasis [19]. The Warburg effect elevates O-GlcNAc levels in cancer cells, and subsequently hyper-O-GlcNAcylation of some proteins enhances the migration/ invasion via modulating
A

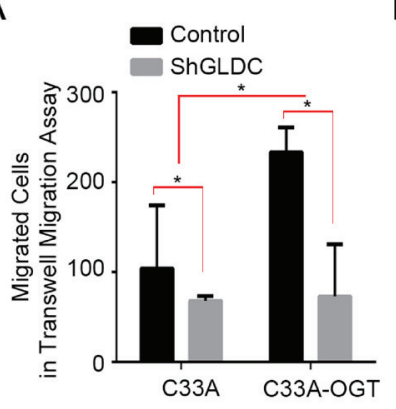

$\mathrm{B}$

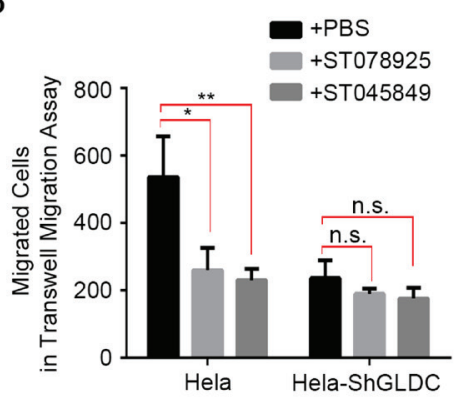

C

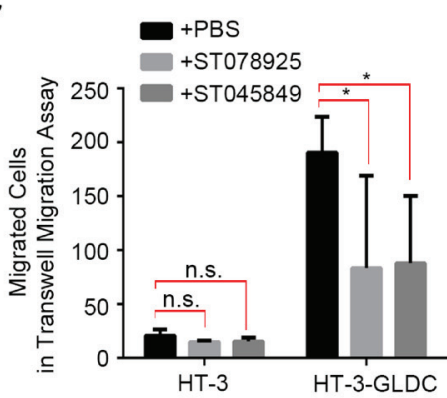

$\mathrm{D}$

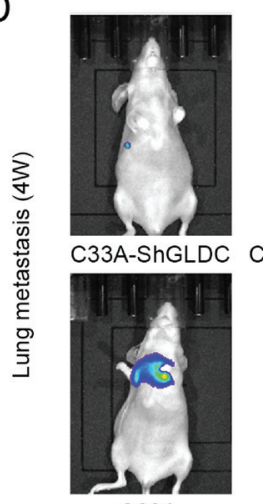

C33A

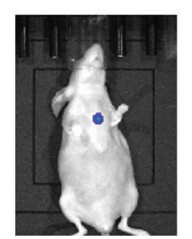

33A-ShGLDC-OGT

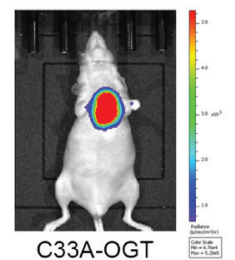

$\mathrm{E}$

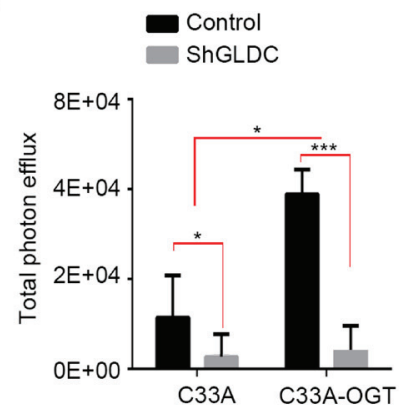

Figure 4: OGT greatly enhances the promoting functions of GLDC on cervical cancer metastasis. (A) $24 \mathrm{~h}$ transwell migration assays in C33A cells subjected to GLDC knockdown and/or OGT overexpression. (B) $24 \mathrm{~h}$ transwell migration assays in Hela cells subjected to GLDC knockdown and/or OGT enzymatic inhibition by ST078925 (50 $\mu \mathrm{M})$ or ST045849 (50 nM). (C) $24 \mathrm{~h}$ transwell migration assays in HT-3 cells subjected to GLDC overexpression and/or OGT enzymatic inhibition by ST078925 (50 $\mu$ M) or Thiamet-G $(20 \mathrm{nM})$. (D-E) In vivo metastasis analysis of intravenous injection and bioluminescent imaging of C33A-luc cells subjected to indicated expression alternations of GLDC and/or OGT. Representative images (D) and statistical data of total photon efflux (E) were shown. n.s., not significant; ${ }^{*} P<0.05 ;{ }^{* *} P<0.01 ;{ }^{* * *} P<0.001$. 
epithelial-to-mesenchymal transition (EMT) [19]. Our present data showed that hyper-O-GlcNAcylation of GLDC could also promote cervical cancer metastasis. In O-GlcNAcylation process, the O-GlcNAc transferase (OGT) transfers the GlcNAc moiety from the high-energy donor UDP-GlcNAc to substrate proteins, whereas O-GlcNAcase (OGA) hydrolyzes O-GlcNAc from proteins [41]. In this study, we detected expression of OGT instead of OGA in cervical cancer, and found that OGT interacted with and modified GLDC with hyper-O-GlcNAcylation in some OGT ${ }^{\text {high }}$ cervical cancer cell lines. And this hyper-OGlcNAcylation on GLDC greatly enhanced the promotion of GLDC on metastasis. Meanwhile, both of our in vitro and in vivo data suggested that the function of OGT on cervical cancer metastasis depended on GLDC attendance. Furthermore, our clinical data revealed the correlation of O-GlcNAcylated GLDC with cervical cancer metastasis and showed that GLDC ${ }^{\text {High }}$ OGT $^{\text {High }}$ patients had worse prognosis. Therefore, O-GlcNAcylation of GLDC might have independent prognostic value in cervical cancer and could provide novel targets for prognostic therapeutics.
Taken together, our data link a glycine metabolism enzyme to cervical cancer and metastasis, and reveal the hyper-O-GlcNAcylation on GLDC and activation on metastasis, which provide a therapeutic target for metastatic cervical cancer and open a new approach for glycine metabolism research. However, the detailed mechanism by which hyper-O-GlcNAcylated GLDC promotes cervical cancer metastasis is still not clear, and would require further investigation.

\section{MATERIALS AND METHODS}

\section{Patients and samples}

This study was approved by the Research Ethics Review Committees of Wenzhou Hospital of Integrated Traditional Chinese and Western medicine (Zhejiang, China). All patients received explanation concerning the aims of the study and provided signed informed consent. Cervical cancer tissues were surgically collected from 146 patients from May 2009 to July 2011 at the Departments
A

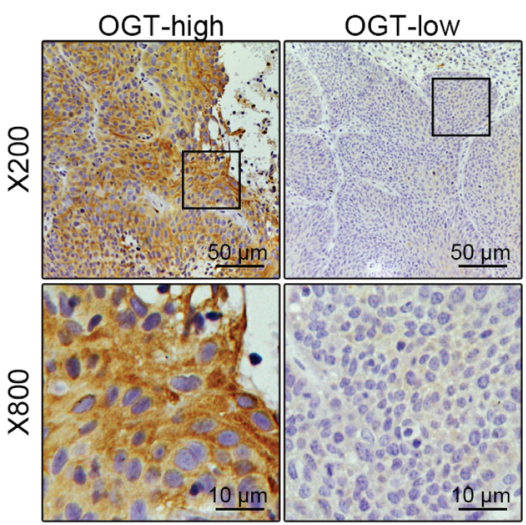

C

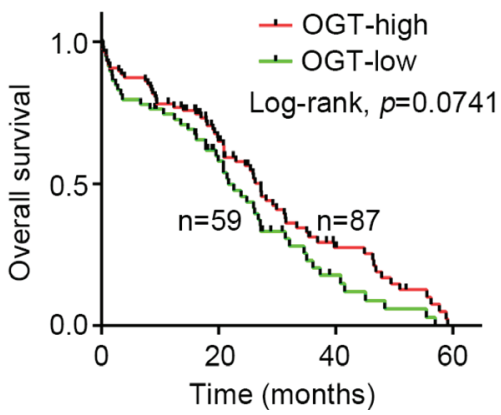

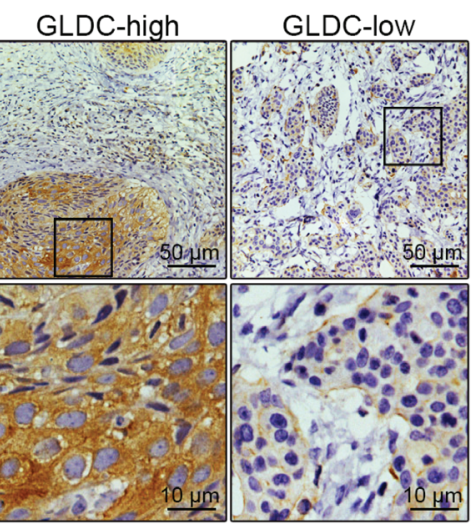

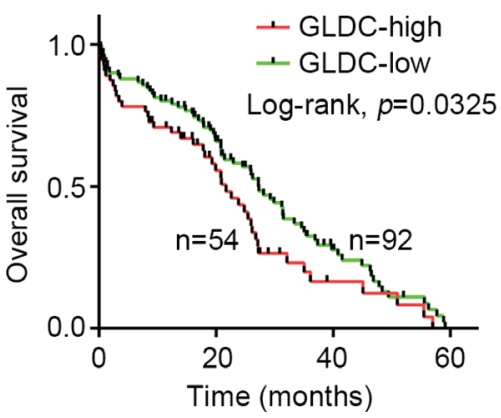

B

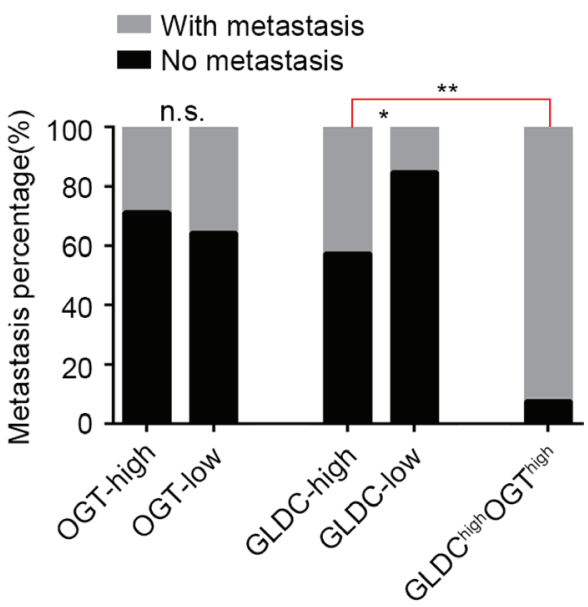

D

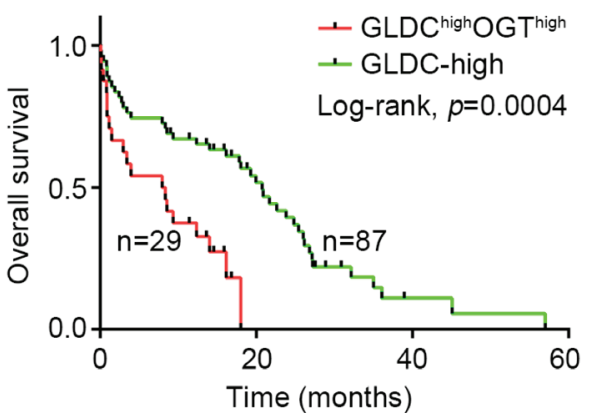

Figure 5: Co-expression of OGT and GLDC is clinically correlates with cervical cancer metastasis and predicts poor prognosis in cervical cancer patients. (A) Representative images of IHC tests stained for OGT and GLDC in human cervical cancer samples. 200× magnification, scale bar $=50 \mu \mathrm{m} ; 800 \times$ magnification, scale bar $=10 \mu \mathrm{m}$. (B) Relative metastatic proportions of cervical cancer patients with different expression of OGT and/or GLDC. (C-D) Overall survival using the Kaplan-Meier method in cervical cancer samples with different expression of OGT and/or GLDC. $P$-values were calculated by the log-rank test. n.s., not significant; ${ }^{*} P<0.05 ;{ }^{* *} P$ $<0.01 ;{ }^{* * *} P<0.001$. 
of Obstetrics and Gynecology, Wenzhou Hospital of Integrated Traditional Chinese and Western medicine (Zhejiang, China). Samples were collected from primary lesions during surgery. All tissue samples were evaluated by three pathologists.

\section{Cell culture}

A normal cervix cell line End1 and four human cervical cancer cell lines, including HT-3, C33A, Caski and HeLa, were purchased from Cell Bank of Type Culture Collection of Chinese Academy of Sciences (Shanghai, China). All cell lines were respectively cultured in complete growth RPMI-1640 medium supplemented with $10 \%$ heat-inactivated fetal bovine serum (Gibco, USA) and $1 \%$ penicillin/streptomycin (Gibco, USA) at $37^{\circ} \mathrm{C}$ in a humidified $5 \% \mathrm{CO}^{2}$ atmosphere.

\section{Quantitative real-time PCR (qRT-PCR)}

Total mRNA was isolated using the Trizol reagent (Qiagen, Valencia, CA, USA). cDNA was synthesized from 200 ng RNA with the PrimeScript RT Reagent Kit (Takara, Dalian, China) according to the manufacturer's instructions. cDNA equivalent to $40 \mathrm{ng}$ total mRNA was used to perform quantitative PCR. PCR reactions processed with SYBR Premix Ex Taq II kit (Takara) and PCR protocol consisted of 1 cycle at $95^{\circ} \mathrm{C}$ for $10 \mathrm{~s}$ followed by 40 cycles at $95^{\circ} \mathrm{C}$ for $5 \mathrm{~s}$ and at $60^{\circ} \mathrm{C}$ for $45 \mathrm{~s}$. The expression of the housekeeping gene GAPDH was used as internal control. Real-time PCR reactions were carried out using Applied Biosystem's 7500 QPCR System (ABI, Foster, CA, USA). The primers were used as followed: GLDC: (5'-CCAGACACGACGACTTCGC-3') (sense) and (5'-CAATTCATCAATGCTCGCCAG-3') (antisense) and GAPDH: (5'-ACCACAGTCCATGCCATCAC-3') (sense) and (5'-TCCACCACCCTGTTGCTGT-3') (antisense).

\section{Plasmid transfection, shRNA and stable cell lines}

For overexpression, full-length GLDC or OGT cDNA was amplified from total mRNA of HeLa cell and cloned into the pCMV-Flag vector (Sigma, USA) and pcDNA3.1 vector (Invitrogen, USA) respectively. For shRNA knock-down, sequences targeting GLDC or OGT were cloned into the pGPH1 vector (GenePharma, China). The shRNA sequences were used as followed: GLDC (5'-gcccatggggctccagttccatct-3') and OGT (5'-ga aaattgtcaagatgaagtgtcct- $\left.3^{\prime}\right)$. Plasmids was transfected into cells using Lipofectamine 3000 (Invitrogen). $48 \mathrm{~h}$ after transfection, cell lysates were prepared for further analysis. For stable cell line selection, transfected cells were treated with $800 \mu \mathrm{g} / \mathrm{mL}$ G418 (Sigma, St. Louis, MO, USA) and the stable transfectants were sustained in the media containing $0.5 \mathrm{mg} / \mathrm{ml} \mathrm{G} 418$. The expression level of GLDC or OGT was confirmed by WB.

\section{Immunoprecipitation (IP) and western blot (WB)}

IP was performed using Protein G IP Kit (Roche, Switzerland) according to the manufacturer's instruction. For western blotting, cell pellets were solubilized with RIPA buffer (Beyotime Institute of Biotechnology, China) with addition of cOmplete Protease Inhibitors (Roche, Switzerland) and PhosSTOP Phosphastase Inhibitors (Roche, Switzerland), electrophoresed, and blotted onto PVDF membranes. The membranes were incubated with indicated primary antibodies followed by the incubation with HRP-conjugated secondary antibodies (Jackson ImmunoResearch, UK). Protein concentration was calculated by the BCA Protein assay kit (Thermo Scientific Pierce, UK). Blotted proteins were visualized using an enhanced chemiluminescence detection kit (Tiangen Biotech, China). The intensity of the bands was analyzed by Quantity One V 4.62 Software. Antibodies against GLDC (Abcam, USA) and myc-tag (Santa Cruz Biotechnology, Santa Cruz, CA, USA) were used for both IP and WB, and antibodies against OGT, phosphorylated serine, phosphorylated threonine, GAPDH, O-GlcNAc (Abcam, USA), GST-tag and Flag-tag (Santa Cruz Biotechnology, Santa Cruz, CA, USA) were used in WB.

\section{GST pull-down}

Full-length GLDC cDNA was cloned into pGEX4T-1 vector and transformed into E.coli. Soluble GST-GLDC was purified on GST-beads from E.coli $8 \mathrm{~h}$ after transformation, and OGT protein was synthesized in TNT reaction with [35S] Methionine. After confirming the positive expression of GST-GLDC and OGT via WB, further pull-down assay was performed with MagneGST Pull-Down system (Promega, WI, USA) according to the manufacturer's instruction. Pull-down result was analyzed with SDS-PAGE and autoradiography.

\section{Cell migration assay}

For migration assays, cells were harvested and suspended in serum free medium supplemented with $1 \%$ BSA. Cell suspension was loaded into the top chamber with a non-coated membrane (Millipore, MA, USA) at a concentration of $1 \times 10^{5}$ cells per $100 \mu \mathrm{L}$. Medium containing $20 \%$ FBS was used as a chemoattractant in the lower chamber. After $24 \mathrm{~h}$ of incubation at $37^{\circ} \mathrm{C}$, cells on the upper surface of the membranes were removed with a cotton swab. The membranes were then stained (Hema3 staining kit; Fisher), and the cells were counted using a 
phase-contrast microscope. Five randomly selected high powered fields were counted for each membrane.

\section{Animal experiments and bioluminescent imaging}

Six-week-old male BALB/c-nu/nu mice were housed at the animal facility of Institute of Wenzhou Medical University (Zhejiang, China). Experiments were performed after approval by the local regulatory authority. For metastasis assay, C33A-luc cells (CellCyto, China) stably expessing OGT and/or shGLDC were suspended in PBS $\left(5^{*} 105 /\right.$ mouse) and injected intravenously into the lateral tail vein of mice. 4 weeks later, bioluminescent imaging was performed with IVIS200 (Xenogen, Caliper, CA, USA) 10 minutes after the intraperitoneal injection of luciferin (3 mg/mouse) (Promega, WI, USA). The intensity of luc-signal was quantified using ROI analysis.

\section{Immunohistochemistry (IHC)}

IHC was performed with UltraVision Quanto Detection System (Thermo Scientific Pierce, UK) according to the manufacturer's instructions. The tissue specimens were fixed overnight in $10 \%$ neutralbuffered formalin and then were dehydrated in increasing concentrations of isopropyl alcohol, followed by clearing of alcohol by xylene. The specimens were subsequently embedded in paraffin wax in cassettes for facilitation of tissue sectioning. Standard staining with hematoxylin and eosin (H\&E) was performed on sections $4 \mu \mathrm{m}$ thickness from each specimen block. For immunohistochemistry, tissue sections were deparaffinized and incubated in citrate buffer at $95^{\circ} \mathrm{C}$ for $40 \mathrm{~min}$ for antigen retrieval and then incubated overnight at $4^{\circ} \mathrm{C}$ with the primary antibodies with anti-GLDC and anti-OGT (Abcam, USA) antibodies in IHC tests.

\section{Statistical analysis}

The statistical analysis were performed with GraphPad Prism v6 (GraphPad Software Inc.). All values were expressed as mean \pm standard error (SE). Two-tailed Student's test was used to assess the statistical significance between two groups. Multiple comparisons were performed by one-way ANOVA test followed by Dunnett's post-hoc analysis (if the ANOVA turned out to be significant). The relationships between clinicopathologic characteristics and GLDC/OGT expression was analyzed by Pearson $\chi^{2}$ test. Survival was evaluated by KaplanMeier survival curves, and the log-rank test was used to evaluate the differences between groups. $P<0.05$ was considered statically significant.

\section{Author contributions}

X.J. performed all the experiments. X.J., P.C., H.Z., S.Z. and L.W. performed the tissues treatments. X.J. and P.C. carried out analysis and interpretation of data; X.J. and L.W. carried out statistical analysis; H.Z. and S.Z. collected clinical data. J.J. wrote the manuscript. J.J. conceived of the idea and designed the experiments. All authors have reviewed the manuscript.

\section{ACKNOWLEDGMENTS AND FUNDING}

This work was supported by grants from the National Natural Science Foundation For Young Scientists of China (31300676), Natural Science Foundation of Zhejiang Province (LY13H070006), Foundation of Science Technology Department of Zhejiang Province (2013C33098), Foundation of Wenzhou Science \&Technology Bureau (Y20140110).

\section{CONFLICTS OF INTEREST}

The authors have declared that no conflicts of interest existed.

\section{REFERENCES}

1. Ferlay J, Soerjomataram I, Dikshit R, Eser S, Mathers C, Rebelo M, Parkin DM, Forman D, Bray F. Cancer incidence and mortality worldwide: sources, methods and major patterns in GLOBOCAN 2012. International journal of cancer. 2015; 136:E359-386.

2. Pfaendler KS, Tewari KS. Changing paradigms in the systemic treatment of advanced cervical cancer. American journal of obstetrics and gynecology. 2016; 214:22-30.

3. Fleming ND, Frumovitz M, Schmeler KM, dos Reis R, Munsell MF, Eifel PJ, Soliman PT, Nick AM, Westin SN, Ramirez PT. Significance of lymph node ratio in defining risk category in node-positive early stage cervical cancer. Gynecologic oncology. 2015; 136:48-53.

4. Sideri M, Igidbashian S. HPV-based screening for prevention of invasive cervical cancer. Lancet. 2014; 383:1294.

5. Kume A, Koyata H, Sakakibara T, Ishiguro Y, Kure S, Hiraga K. The glycine cleavage system. Molecular cloning of the chicken and human glycine decarboxylase cDNAs and some characteristics involved in the deduced protein structures. The Journal of biological chemistry. 1991; 266:3323-3329.

6. Go MK, Zhang WC, Lim B, Yew WS. Glycine decarboxylase is an unusual amino acid decarboxylase involved in tumorigenesis. Biochemistry. 2014; 53:947-956. 
7. Kim D, Fiske BP, Birsoy K, Freinkman E, Kami K, Possemato RL, Chudnovsky Y, Pacold ME, Chen WW, Cantor JR, Shelton LM, Gui DY, Kwon M, et al. SHMT2 drives glioma cell survival in ischaemia but imposes a dependence on glycine clearance. Nature. 2015; 520:363-367.

8. Tibbetts AS, Appling DR. Compartmentalization of Mammalian folate-mediated one-carbon metabolism. Annual review of nutrition. 2010; 30:57-81.

9. Zhang WC, Shyh-Chang N, Yang H, Rai A, Umashankar S, Ma S, Soh BS, Sun LL, Tai BC, Nga ME, Bhakoo KK, Jayapal SR, Nichane M, et al. Glycine decarboxylase activity drives non-small cell lung cancer tumor-initiating cells and tumorigenesis. Cell. 2012; 148:259-272.

10. Noh S, Kim do H, Jung WH, Koo JS. Expression levels of serine/glycine metabolism-related proteins in triple negative breast cancer tissues. Tumour biology. 2014; 35:4457-4468.

11. Kim YH, Jung WH, Koo JS. Expression of metabolismrelated proteins in invasive lobular carcinoma: comparison to invasive ductal carcinoma. Tumour biology. 2014; 35:10381-10393.

12. Kwon JE, Kim DH, Jung WH, Koo JS. Expression of serine and glycine-related enzymes in phyllodes tumor. Neoplasma. 2014; 61:566-578.

13. Sun WY, Kim HM, Jung WH, Koo JS. Expression of serine/ glycine metabolism-related proteins is different according to the thyroid cancer subtype. Journal of translational medicine. 2016; 14:168.

14. Kim HM, Jung WH, Koo JS. Site-specific metabolic phenotypes in metastatic breast cancer. Journal of translational medicine. 2014; 12:354.

15. Yang X, Qian K. Protein O-GlcNAcylation: emerging mechanisms and functions. Nature reviews Molecular cell biology. 2017; 18:452-465.

16. Chen Q, Chen Y, Bian C, Fujiki R, Yu X. TET2 promotes histone O-GlcNAcylation during gene transcription. Nature. 2013; 493:561-564.

17. Jang H, Kim TW, Yoon S, Choi SY, Kang TW, Kim SY, Kwon YW, Cho EJ, Youn HD. O-GlcNAc regulates pluripotency and reprogramming by directly acting on core components of the pluripotency network. Cell stem cell. 2012; 11:62-74.

18. Hanover JA, Krause MW, Love DC. Bittersweet memories: linking metabolism to epigenetics through O-GlcNAcylation. Nature reviews Molecular cell biology. 2012; 13:312-321.

19. Ma Z, Vosseller K. Cancer metabolism and elevated O-GlcNAc in oncogenic signaling. The Journal of biological chemistry. 2014; 289:34457-34465.

20. Buren S, Gomes AL, Teijeiro A, Fawal MA, Yilmaz M, Tummala KS, Perez M, Rodriguez-Justo M, Campos-Olivas R, Megias D, Djouder N. Regulation of OGT by URI in Response to Glucose Confers c-MYC-Dependent Survival Mechanisms. Cancer cell. 2016; 30:290-307.
21. Slawson C, Hart GW. O-GlcNAc signalling: implications for cancer cell biology. Nature reviews Cancer. 2011; 11:678-684.

22. Wang T, Yu Q, Li J, Hu B, Zhao Q, Ma C, Huang W, Zhuo L, Fang H, Liao L, Eugene Chin Y, Jiang Y. O-GlcNAcylation of fumarase maintains tumour growth under glucose deficiency. Nature cell biology. 2017; 19:833-843.

23. Swamy M, Pathak S, Grzes KM, Damerow S, Sinclair LV, van Aalten DM, Cantrell DA. Glucose and glutamine fuel protein O-GlcNAcylation to control T cell self-renewal and malignancy. Nature immunology. 2016; 17:712-720.

24. Rao X, Duan X, Mao W, Li X, Li Z, Li Q, Zheng Z, Xu H, Chen M, Wang PG, Wang Y, Shen B, Yi W. O-GlcNAcylation of G6PD promotes the pentose phosphate pathway and tumor growth. Nature communications. 2015; 6:8468.

25. Kim M, Kim YS, Kim H, Kang MY, Park J, Lee DH, Roh GS, Kim HJ, Kang SS, Cho GJ, Park JK, Cho JW, Shin JK, et al. O-linked N-acetylglucosamine transferase promotes cervical cancer tumorigenesis through human papillomaviruses E6 and E7 oncogenes. Oncotarget. 2016; 7:44596-44607. http://doi.org/10.18632/oncotarget.10112.

26. Zeng Q, Zhao RX, Chen J, Li Y, Li XD, Liu XL, Zhang WM, Quan CS, Wang YS, Zhai YX, Wang JW, Youssef M, Cui R, et al. O-linked GlcNAcylation elevated by HPV E6 mediates viral oncogenesis. Proceedings of the National Academy of Sciences of the United States of America. 2016; 113:9333-9338.

27. Kim S, Shah K. Dissecting yeast Hog1 MAP kinase pathway using a chemical genetic approach. FEBS letters. 2007; 581:1209-1216.

28. Zhu B, Xiahou Z, Zhao H, Peng B, Zhao H, Xu X. MTHFR promotes heterochromatin maintenance. Biochemical and biophysical research communications. 2014; 447:702-706.

29. Yamada K, Strahler JR, Andrews PC, Matthews RG. Regulation of human methylenetetrahydrofolate reductase by phosphorylation. Proceedings of the National Academy of Sciences of the United States of America. 2005; 102:10454-10459.

30. Berezowska S, Galvan JA, Langer R, Bubendorf L, Savic S, Gugger M, Schmid RA, Marti TM. Glycine decarboxylase and HIF-1alpha expression are negative prognostic factors in primary resected early-stage non-small cell lung cancer. Virchows Arch. 2017; 470:323-330.

31. Min HL, Kim J, Kim WH, Jang BG, Kim MA. Epigenetic Silencing of the Putative Tumor Suppressor Gene GLDC (Glycine Dehydrogenase) in Gastric Carcinoma. Anticancer research. 2016; 36:179-187.

32. Li X, Cui C, Guo Y, Yang G. Glycine Decarboxylase Expression Increased in p53-Mutated B Cell Lymphoma Mice. Oncology research and treatment. 2015; 38:586-589.

33. Noh S, Kim DH, Jung WH, Koo JS. Expression levels of serine/glycine metabolism-related proteins in triple negative breast cancer tissues. Tumour biology. 2014; $35: 4457-4468$. 
34. Huang R, Rofstad EK. Cancer stem cells (CSCs), cervical CSCs and targeted therapies. Oncotarget. 2017; 8:3535135367. http://doi.org/10.18632/oncotarget.10169.

35. Todaro M, Gaggianesi M, Catalano V, Benfante A, Iovino F, Biffoni M, Apuzzo T, Sperduti I, Volpe S, Cocorullo G, Gulotta G, Dieli F, De Maria R, et al. CD44v6 is a marker of constitutive and reprogrammed cancer stem cells driving colon cancer metastasis. Cell stem cell. 2014; 14:342-356.

36. Chen D, Wu M, Li Y, Chang I, Yuan Q, Ekimyan-Salvo M, Deng P, Yu B, Yu Y, Dong J, Szymanski JM, Ramadoss S, Li J, et al. Targeting BMI1+ Cancer Stem Cells Overcomes Chemoresistance and Inhibits Metastases in Squamous Cell Carcinoma. Cell stem cell. 2017; 20:621-634 e626.

37. Pollari S, Kakonen SM, Edgren H, Wolf M, Kohonen P, Sara H, Guise T, Nees M, Kallioniemi O. Enhanced serine production by bone metastatic breast cancer cells stimulates osteoclastogenesis. Breast cancer research and treatment. 2011; 125:421-430.
38. Liu B, Jia Y, Cao Y, Wu S, Jiang H, Sun X, Ma J, Yin X, Mao A, Shang M. Overexpression of Phosphoserine Aminotransferase 1 (PSAT1) Predicts Poor Prognosis and Associates with Tumor Progression in Human Esophageal Squamous Cell Carcinoma. Cellular physiology and biochemistry. 2016; 39:395-406.

39. Liao KM, Chao TB, Tian YF, Lin CY, Lee SW, Chuang HY, Chan TC, Chen TJ, Hsing $\mathrm{CH}$, Sheu MJ, Li CF. Overexpression of the PSAT1 Gene in Nasopharyngeal Carcinoma Is an Indicator of Poor Prognosis. Journal of Cancer. 2016; 7:1088-1094.

40. Weber GF. Metabolism in cancer metastasis. International journal of cancer. 2016; 138:2061-2066.

41. Wells L, Vosseller K, Hart GW. Glycosylation of nucleocytoplasmic proteins: signal transduction and O-GlcNAc. Science. 2001; 291:2376-2378. 